\title{
Neurorehabilitation - To Facilitate or to Impede
}

\section{Eli Carmeli ${ }^{*}$}

Physical Therapy Department, University of Haifa, Israel

*Corresponding author: Carmeli E, Physical Therapy Department, University of Haifa, Israel, Tel: +972 48288397; Fax: +972 48288140; E-mail: ecarmeli@univ.haifa.ac.il

Received date: June 22, 2015; Accepted date: June 24, 2015; Published date: June 30, 2015

Copyright: @ 2015 Carmeli E, et al. This is an open-access article distributed under the terms of the Creative Commons Attribution License, which permits unrestricted use, distribution, and reproduction in any medium, provided the original author and source are credited.

\section{Editorial}

Brain disorders resulting from pathological conditions such as CVA, Parkinson, Down's syndrome, traumatic brain injury, and $\mathrm{CP}$ are common. These pathologies, frequently effects the function of upper and lower limbs causing spasticity, atrophy and difficulties to move hand and leg, thus affecting the upper limb trajectories and ability to grasp, grip, reach, manipulate objects, and affecting the lower limb trajectories and ability to step up and down, walk, and kick a ball.

Motor learning and motor control are the two main mechanisms responsible for enhanced functional performance after a stroke [1]. In contrast, increased use of compensation strategies is characterized by using different movement patterns than normal to facilitate accomplishment of daily motor tasks. Therefore, overall improvements in motor performance should be attributed to a combination of these two mechanisms [2].

Traditionally, therapy to improve movements and functions mainly used 'facilitation approach' such as neuro-developmental treatment (NDT) which strives to facilitate normal movements through the use of therapeutic handling. The recovery of movement patterns toward the pre-injury state, manifested by increased ability to isolate the movements of the hand, forearm, arm, hip, knee and ankle, accompanied by various structural and physiological changes within the brain. Therefore, NDT emphasizing a great deal of training time, repetitions, lot of handling to reduce muscle tone, and guidance to produce a desired movement, that can directly result to better performance in activities of daily living, and indirectly lead to increase social integration and can thus produce a better quality of life.

For the last few decades, a task-oriented exercise approach [3] has been recommended over neuro developmental therapy to facilitate motor recovery. This approach is based on a systems model of motor behavior that integrates factors related to the client, the task and the environment and their influence on motor performance.

Moreover, researchers have recently begun to investigate a novel application of robotics $[4,5]$ as a potential modality to enhance neuro rehabilitation that leverages the power of neuro-plasticity but using error augmentation approach [6].

In this paradigm the robotic computer singles out and magnifies the subject's movement errors from a desired trajectory, alters the visual feedback of the movement trajectory, hence emphasizing the visual and sensory feedback, and utilizes erroneous feedback to enhance motor recovery, and consequently forcing the patients to strengthen their motor control. This feedback is counterintuitive and differs greatly from the standard level of care such as NDT. In addition to the above scientific justifications for applying error augmentation approach, robotic-assisted training can be highly accurate, sustained for very long periods of time, measure progress automatically, produce a wide range of forces or motions, and offers exciting new prospects for the regaining motor control after neural injury [7].

\section{References}

1. Castro LNG, Monsen CB, Smith MA (2011) The binding of learning to action in motor adaptation. PLoS Comput Biol 7: e1002052.

2. Taylor JA, Ivry RB (2012) The role of strategies in motor learning. Ann N Y Acad Sci 1251: 1-12.

3. Molier BI, Prange GB, Krabben T, Stienen A, van der Kooij H, et al. (2011) Effect of position feedback during task-oriented upper-limb training after stroke: Five-case pilot study. J Rehabil Res Dev 48: 1109-1118.

4. Givon-Mayo R, Simons E, Ohry A, Karpin H, Israely S, et al. (2014) A preliminary investigation of error enhancement of the velocity component in stroke patients' reaching movements. Int J Ther Rehabil 21: 160-168.

5. Huang V, Krakauer J (2009) Robotic neurorehabilitation: A computational motor learning perspective. J Neuroeng Rehabil 6: 5.

6. Alexoulis-Chrysovergis AC, Weightman A, Hodson-Tole EF, Deconinck FJ (2013) Error augmented robotic rehabilitation of the upper limb - A review. Neurotechnix - International congress on neurotechnology, electronics and informatics 167-178.

7. Patton JL, Stoykov ME, Kovic M, Mussa-Ivaldi FA (2006) Evaluation of robotic training forces that either enhance or reduce error in chronic hemiparetic stroke survivors. Exp Brain Res 168: 368-383. 